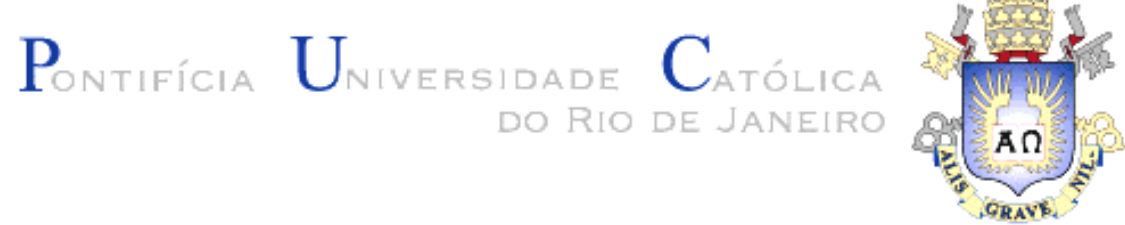

Mariana da Costa Novo Pimenta Brandão

Modelagem da Eletrodinâmica Cardíaca e dos Efeitos da Resolução Espacial e Temporal em Mapas Ópticos.

Tese de Doutorado

Tese apresentada ao Programa de Pós-graduação em Física da PUC-Rio como requisito parcial para obtenção do título de Doutor em Física.

Orientadores: Isabel Cristina dos Santos Carvalho Elisabeth Costa Monteiro

Rio de Janeiro, fevereiro de 2011 


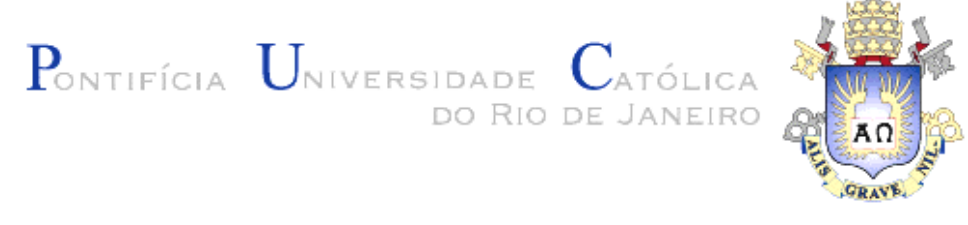

Mariana da Costa Novo Pimenta Brandão

\title{
Modelagem da Eletrodinâmica Cardíaca e dos Efeitos da Resolução Espacial e Temporal em Mapas Ópticos.
}

\begin{abstract}
Tese apresentada como requisito parcial para obtenção do título de Doutor pelo Programa de Pós-Graduação em Física da PUC-Rio. Aprovada pela Comissão Examinadora abaixo assinada.
\end{abstract}

Prof. Isabel Cristina dos Santos Carvalho
Orientador
Departamento de Física - PUC-Rio

Prof. Elisabeth Costa Monteiro Orientador Departamento de Metrologia - PUC-Rio

Prof. Marcio Nogueira de Souza UFRJ

Prof. Daniel Acosta Avalos CBPF

Prof. Carlos Roberto Hall Barbosa Departamento de Metrologia - PUC-Rio

Prof. Welles Antônio Martinez Morgado Departamento de Física - PUC-Rio

Prof. Jorge Luis Gonzalez Alfonso UFES

Prof. José Eugênio Leal Coordenador(a) Setorial do Centro Técnico Científico - PUC-Rio 
Todos os direitos reservados. É proibida a reprodução total ou parcial do trabalho sem autorização da universidade, da autora e do orientador.

\section{Mariana da Costa Novo Pimenta Brandão}

Graduou-se Física pela Universidade Federal de Juiz de Fora-UFJF em 2004. É mestre em física na área de Óptica pela Universidade Federal de Juiz de Fora-UFJF em 2006 e doutora em física pela PUC-Rio na área de Biofísica.

Ficha Catalográfica

Brandão, Mariana da Costa Novo Pimenta

Modelagem da eletrodinâmica cardíaca e dos efeitos da resolução espacial e temporal em mapas ópticos / Mariana da Costa Novo Pimenta Brandão ; orientadoras: Isabel Cristina dos Santos Carvalho, Elisabeth Costa Monteiro. 2011.

116 f. : il.(color.) ; $30 \mathrm{~cm}$

Tese (doutorado)-Pontifícia Universidade Católica do Rio de Janeiro, Departamento de Física, 2011.

Inclui bibliografia

1. Física - Teses. 2. Potencial de ação óptico. 3. Modelagem matemática. 4. Resolução espacial e temporal. 5. Mapeamento óptico. 6. Processamento de sinais. I. Carvalho, Isabel Cristina dos Santos. II. Monteiro, Elisabeth Costa. III. Pontifícia Universidade Católica do Rio de Janeiro. Departamento de Física. III. Título.

CDD: 530 
Dedico esta tese à Ruth Costa Novo Pimenta (in memoriam). 


\section{Agradecimentos}

À Dra. Isabel Cristina dos Santos Carvalho e Dra. Elisabeth Costa Monteiro pela orientação, presença, compreensão e amizade;

Ao Dr. Eduardo Andrade de Lima pela paciência, generosidade e colaboração de extrema pertinência;

À Dra. Fátima Gunning e Dr. Andrew Ellis pela oportunidade e orientação durante estágio no Tyndall National Institute;

Ao Dr. Jorge Luis Gonzalez Alfonso pelas conversas e ajuda nos problemas de programação;

Ao Dr. Carlos Roberto Hall Barbosa pela ajuda com a modelagem da propagação da atividade elétrica;

Ao Dr. John P. Wikswo pela disponibilizar a infra-estrutra para medições de mapas ópticos em corações isolados no Viibre;

À Dra. Luciana Garzoni pela colaboração e contribuição para este trabalho;

À Pontifícia Universidade Católica do Rio de Janeiro, pela infraestrutura para realização do curso de doutorado;

A CAPES e CNPq por fornecer a bolsa de estudos que possibilitou a realização deste trabalho;

Ao Dr. Hélio Ricardo pela disposição e ajuda durante este trabalho;

Aos alunos da sala 656L por tornar os dias mais agradáveis;

Aos amigos do Laboratório de Optoeletrônica pelo apoio e risadas;

Aos meus familiares, em especial aos meus pais e irmão Gabriel, pelo apoio em todas as horas.

A todos os amigos pela paciência em todos os dias que não pude comparecer, em particular aos atletas de mesa pela disposição em tornar os dias mais alegres;

Em especial, à Rian, Blau e Paula por todos os momentos divertidos que ajudam a manter a mente sã;

E ao Otto porque o sol já nasceu. 


\section{Resumo}

Brandão, M.P.; Carvalho, I.C.S. Modelagem da Eletrodinâmica Cardíaca e dos Efeitos da Resolução Espacial e Temporal em Mapas Ópticos. Rio de Janeiro, 2011. 116p. Tese de Doutorado - Departamento de Física, Pontifícia Universidade Católica do Rio de Janeiro.

A detecção da fluorescência de corantes poteciométricos aplicados em tecidos excitáveis vem sendo utilizada não somente para a realização de estudos da propagação da frente de onda da excitação elétrica tecidual, como também na análise dos diferentes parâmetros eletrofisiológicos caracterizados pela morfologia do potencial de ação óptico. Apesar dos avanços no desenvolvimento de sistemas de detecção, o compromisso entre taxa de amostragem temporal, sensibilidade, resolução espacial e campo de visão na técnica de mapeamento óptico frequentemente acarreta um potencial de ação medido experimentalmente que não corresponde à resposta elétrica de uma única célula, mas à soma da atividade elétrica de várias células adjacentes. Consequentemente, a análise e interpretação de estudos quantitativos de parâmetros eletrofisiológicos da ativação elétrica tecidual, comparando o potencial de ação óptico com o potencial de ação de uma única célula, como o obtido por medição intracelular utilizando microeletrodo, podem ser afetadas pelo efeito de integração espacial do detector óptico. Adicionalmente, o potencial de ação óptico e a visualização da propagação da atividade elétrica podem ser distorcidos por mapas ópticos cuja resolução temporal da aquisição seja inadequada. Neste trabalho, por meio de simulações matemáticas, mapeamento óptico e processamento de sinais, foram estudados os impactos na configuração do potencial de ação óptico associados à resolução espacial e temporal utilizada na detecção da fluorescência em resposta à ativação elétrica tecidual em corações isolados.

\section{Palavras-chave}

Potencial de Ação Óptico; Modelagem Matemática; Resolução Espacial e Temporal; Mapeamento Óptico; Processamento de Sinais. 


\section{Abstract}

Brandao, M.P.; Carvalho, I.C.S. (Advisor). Modeling of the Cardiac Electrodynamics and of the Effects of Spatial and Time Resolution on Optical Maps. Rio de Janeiro, 2011. 116p. PhD Thesis - Departamento de Física, Pontifícia Universidade Católica do Rio de Janeiro.

Fluorescence studies using potentiometric dyes to optically measure the action potential of cardiac cells constitute an important tool to better understand the electrophysiology of the heart. However, in many experimental setups, the finite sensing area of optical detectors cannot be neglected. As a consequence, the detected fluorescence signal may effectively correspond to the sum of the electrical activity of a group of adjacent cells, and variations observed in the fluorescence signal may be strongly influenced by the spatial resolution of the optical instrumentation employed in the experiment. In addition, the optical action potential and the visualization of its propagation may be distorted by inadequate temporal resolution when recording optical maps. In this work, we present simulated and experimental studies demonstrating the effects of both temporal and spatial resolutions of the fluorescence detection system on electrophysiological parameter estimates obtained from optical action potential recordings. The analysis of the influence of the spatial and temporal resolutions of the optical fluorescence mapping detection system on the optical action potential morphology were performed by means of experimental optical mapping recordings, action potential simulations, and digital processing of the simulated and experimental signals.

\section{Keywords}

Optical Action Potential; Mathematical Model; Spatial and Temporal Resolution; Optical Mapping; Signal Processing. 


\section{Sumário}

1. Introdução

2. Medição Óptica da Atividade Elétrica Celular 22

2.1. Eletrofisiologia Celular ${ }^{[25-29]} 22$

2.1.1.Cardiomiócito 24

2.1.2. Correntes Transmembranares e Modelagem Matemática 27

2.2. Mapas ópticos e sua instrumentação 31

2.2.1. Corantes Potenciométricos [19,47-49] 31

2.2.2. Sensores CCD 33

2.3. Processamento de Sinais 36

2.3.1. Filtro Butterworth 36

2.3.2. Interpolação por Cubic Spline e Spline Fit 36

3. Metodologia 38

3.1.Mapeamento óptico da fluorescência gerada pela atividade elétrica de corações isolados 38

3.1.1. Preparação do coração para mapeamento óptico 39

3.1.2. Montagem Experimental 40

3.2. Simulação da resposta elétrica de células cardíacas 42

3.2.1. Simulação de uma célula ventricular do coração de coelho 43

3.2.2. Simulação de um grupo de 125 células ventriculares do coração de coelho 53

3.3. Processamento de sinais experimentais e simulados 54

3.3.1. Filtragem do Ruído por Filtros Butterworth. 55

3.3.2. Redução da resolução espacial por binagem 56

3.4. Indicadores de distorção do potencial de ação 57

4. Resultados 60

4.1 Simulação da resposta elétrica de células cardíacas 60

4.1.1.Simulação de uma célula ventricular do coração de coelho 60 
4.1.2. Simulação de um grupo de 125 células ventriculares do coração de coelho

4.2. Mapeamento óptico da fluorescência gerada pela atividade elétrica de corações isolados

65

4.2.1.Resultados Experimentais

66

4.3. Análise dos efeitos do processamento digital na morfologia de sinais experimentais e simulados

69

4.3.1 Filtragem do Ruído e Melhora da resolução temporal 69

4.3.1.1. Cubic Spline, Spline Fit e Butterworth 69

4.3.1.2. Potenciais de ação ópticos 73

4.4. Análise qualitativa e quantitativa da influência das resoluções espaciais e temporais. $\quad 74$

4.4.1. Propagação da Atividade Elétrica $\quad 74$

4.4.2. Parâmetros do upstroke do potencial de ação 80

4.4.2.1. Parâmetros do upstroke do potencial de ação simulado 80

4.4.2.2. Parâmetros do upstroke do potencial de ação ótico 84

5. Discussão e Conclusões 89

5.1. Influência da Resolução Espacial e Temporal em mapeamentos ópticos da atividade cardíaca 89

5.2. Conclusões 98

5.3. Perspectivas e Trabalhos Futuros 99

6. Referências Bibliográficas 102

$\begin{array}{ll}\text { Apêndice } 1 & 109\end{array}$ 


\section{Lista de Figuras:}

Figura 1: Potencial de ação cardíaco mostrando cada fase: (4) Repouso, (0) Despolarização, (1) Repolarização Inicial, (2) Platô, (3) Repolarização, e as correntes iônicas relacionadas com a mudança de potencial em cada fase.

Figura 2: Esquema da contração cardíaca, potenciais de ação de cada tipo celular e resposta resultante de eletrocardiograma.

Figura 3: Representação da modelagem matemática do potencial de ação por Hodgkin e Huxley, possuindo canais de sódio e potássio e uma corrente de vazamento, que inclui os outros canais não descritos explicitamente.

Figura 4: Absorção e Emissão do corante di-4-ANEPPS: a carga desloca-se por ressonância, para o final da molécula e se localiza no nitrogênio da anilina.

Figura 5: Orientação das Moléculas de ANEP no interior da membrana celular.

Figura 6: Detecção do potencial de ação de um coração isolado de coelho corado com Di-4-ANEPPS. A excitação foi feita com um laser de argônio operando em 488nm e a detecção foi feita simultaneamente com fotomultiplicadoras em $540 \mathrm{~nm}$ e $610 \mathrm{~nm}$ (A). Razão entre as duas curvas de fluorescência (B). Um microeletrodo intracelular foi utilizado simultaneamente às medidas $(\mathrm{C})$.

Figura 7: Esquema de um sensor CCD. 34

Figura 8: Representação do processo de binagem 2x2 de um sensor CCD. 35

Figura 9: Esquema da montagem experimental para mapeamento óptico 41

Figura 10: Coração isolado de coelho fixo em sistema Langerdoff na montagem experimental para imageamento óptico de sua atividade elétrica.

Figura 11: Representação das correntes iônicas que compõem a modelagem utilizada para o cálculo do potencial de ação de uma célula ventricular de coração de coelho.

Figura 12: Esquema da distribuição do grupo de 125 células para cálculo do potencial de ação médio gerado $\left(\delta_{\mathrm{L}}\right.$ e $\left.\delta_{\mathrm{T}} \mathrm{em} \mathrm{ms}\right)$. 
Figura 13: Representação dos parâmetros $\mathbf{T}_{\mathbf{S}(\max )}, \mathbf{V}_{\mathbf{S}(\max )}$ e $\mathbf{V}_{\mathbf{S}}{ }^{*}$ para o upstroke de um potencial de ação médio simulado para um grupo de 125 células.

Figura 14: Potencial de ação e correntes iônicas gerados por meio de modelagem matemática. $\mathrm{I}_{\mathrm{Na}}$ : Corrente de sódio rápida; $\mathrm{I}_{\mathrm{Kr}}$ : Corrente de potássio ativada rapidamente; $\mathrm{I}_{\mathrm{Ks}}$ : Corrente de potássio ativada lentamente; $\mathrm{I}_{\mathrm{Kp}}$ : Corrente de potássio de Plateau; $\mathrm{I}_{\mathrm{k} 1}$ : Corrente de potássio independente do tempo; $\mathrm{I}_{\mathrm{Ca}, \mathrm{L}}$ : Corrente de cálcio que atravessa canais do tipo-L; $\mathrm{I}_{\mathrm{Ca}, \mathrm{T}}$ : Corrente de cálcio que atravessa canais do tipo-T; $\mathrm{I}_{\mathrm{Na}, \mathrm{b}}$ : Corrente de fundo de sódio; $\mathrm{I}_{\mathrm{Ca}, \mathrm{b}}$ : Corrente de fundo de cálcio; $\mathrm{I}_{\mathrm{pCa}}$ : Corrente da bomba de cálcio do retículo sarcoplasmático; $\mathrm{I}_{\mathrm{NaCaX}}$ : Fluxo pelo trocador sódio-cálcio; $\mathrm{I}_{\mathrm{Na}, \mathrm{K}}$ : Corrente da bomba sódio-potássio; $\mathrm{I}_{\mathrm{to}, \mathrm{f}}$ : Corrente transiente de saída rápida e $\mathrm{I}_{\mathrm{to}, \mathrm{s}}$ : Corrente transiente de saída lenta.

Figura 15: Comparação da forma do potencial de ação de uma única célula e do potencial de ação médio de um grupo de 125 células cardíacas.

Figura 16: Derivada no tempo dos potenciais de ação simulados para uma célula, $\mathrm{AP}_{\text {cell, }}$ e para o $\mathrm{AP}_{\mathrm{avg}}$ de 125 células. Os efeitos da integração espacial na morfologia do sinal são mais evidentes nas regiões do upstroke e da repolarização do potencial de ação.

Figura 17: Comparação da forma dos upstrokes dos potenciais de ação para uma célula e para o grupo de 125 células.

Figura 18: Mapas ópticos da ativação do tecido cardíaco com formas geométricas para indicando 10 pixels. Vermelho representa excitação e azul representa repouso ou em estágio de repolarização.

Figura 19: Potenciais de ação ópticos para os 10 pixels marcados na sequência de mapas de ativação apresentada na Figura 18. As linhas A e Q mostram o primeiro e o último mapa da sequência.

Figura 20: Potenciais de ação simulados para um grupo de 125 células, $\mathrm{AP}_{\text {avg }}$, com resolução temporal de $2,0 \mathrm{~ms}$ e processados com resoluções temporais de $0,1 \mathrm{~ms}, 0,2 \mathrm{~ms}, 0,3 \mathrm{~ms}, 0,4 \mathrm{~ms}, 0,5 \mathrm{~ms}$ e $1,0 \mathrm{~ms}$ por meio de Cubic Spline (A) e Spline Fit (B) e suas respectivas derivadas temporais (C e D).

Figura 21: Potenciais de ação médios simulados com resolução temporal de $0,1 \mathrm{~ms}$ e $2,0 \mathrm{~ms}$, com adição de ruído e resolução temporal de $2,0 \mathrm{~ms}$ e 
processamento digital por Butterworth, com duas frequências de corte $(75 \mathrm{~Hz}$ e $88 \mathrm{~Hz})$ e Spline Fit com resolução temporal final de 0,1ms (A); e suas respectivas derivadas (B).

Figura 22: Ampliação dos sinais nos upstrokes dos potenciais de ação médios simulados com resolução temporal de $0,1 \mathrm{~ms}$ e $2,0 \mathrm{~ms}$, com adição de ruído e resolução temporal de 2,0ms e processados digital por Butterworth com duas frequências de corte $(75 \mathrm{~Hz}$ e $88 \mathrm{~Hz})$ e Spline Fit com resolução temporal final de $0,1 \mathrm{~ms}$.

Figura 23: Potencial de ação óptico registrado pelos pixels + e $\mathbf{O}$, antes e depois do processamento digital por Butterworth e Spline Fit.

Figura 24: Ativação elétrica do coração isolado de coelho, em determinado instante, para as resoluções espaciais de 0,4mm (128x128 pixels por quadro), 0,8mm (64x64 pixels por quadro), 1,2mm $(32 \times 32$ pixels por quadro) e $3,2 \mathrm{~mm}$ (16x16 pixels por quadro).

Figura 25: Potenciais de ação ópticos registrado pelos pixels + (esquerda) e O (direita) e processados por binagem para reduzir a resolução espacial em combinações de $2 \times 2,3 \times 3,4 \times 4$ e $8 \times 8$.

Figura 26: Comparação de uma sequência de frames de 953,4ms (I) a 1024,62ms (Q), com intervalo entre eles de 10,18ms, para mapas ópticos com diferentes resoluções espaciais: 128x128 pixels, 64x64 pixels, 32x32 pixels e 16x16 pixels.

Figura 27: Mapa de ativação no instante 967,57ms (entre os instantes J e L), com resolução espacial de $0,4 \mathrm{~mm}$ (128x128 pixels) e $3,2 \mathrm{~mm}$ (16x16 pixels) com indicação da posição do pixel \# (seta) onde ocorre um bloqueio visualizado somente no mapa com maior resolução espacial (128x128 pixels).

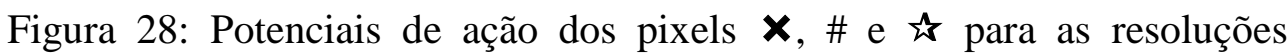
espaciais de $0,4 \mathrm{~mm}$ (A) e 3,2mm (B), com a indicação dos instantes de A a Q. Os pixel $\mathbf{X}$ e o pixel B (onde ocorre um bloqueio de condução) são individualmente visualizados somente no registro com maior resolução espacial $(0,4 \mathrm{~mm})$

Figura 29: Duração do upstroke de potenciais de ação, medidos por meio de mapeamento óptico (linhas sólidas) e por meio de microeletrodos (linha tracejada), como função da velocidade de condução da frente de onde plana. 
A variação da velocidade se deve à variação do acoplamento elétrico das células na direção de propagação. A duração do upstroke do potencial de ação óptico para uma onda plana se afastando (linha preta, circulo sólido) e perpendicular (linha cinza, quadrado sólido) à superfície do tecido, e dos potenciais de ação medido eletricamente (linha tracejada), que foram iguais para os dois casos(onda plana se afastando e perpendicular a superfície). Figura adaptada da referência 17.

Figura 30: Esquema da montagem experimental da técnica de medição óptica na PUC-Rio. 


\section{Lista de Tabelas:}

Tabela 1: Parâmetros $\mathrm{T}_{\mathrm{S}(\max ),} \mathrm{V}_{\mathrm{S}(\max )}$ e $\mathrm{V}_{\mathrm{S}} *$ calculados para potenciais simulados $\mathrm{AP}_{\text {cell }}$ e de um grupo de 125 células, $\mathrm{AP}_{\mathrm{avg}}$, com resoluções temporais de $0,1 \mathrm{~ms}, 0,2 \mathrm{~ms}, 0,3 \mathrm{~ms}, 0,4 \mathrm{~ms}, 0,5 \mathrm{~ms}, 1,0 \mathrm{~ms}$ e $2,0 \mathrm{~ms}$.

Tabela 2: Parâmetros $T_{S(\max )}, V_{S(\max )}$ e $V_{S} *$ calculados para potenciais $A_{\text {avg }}$ de 125 células, simulados com resoluções temporais de $0,1 \mathrm{~ms}$ a 2,0ms, esse último com adição de ruído e submetido à aplicação dos filtros Butterworth (or.4 Fc 88Hz) e Spline Fit, interpolados para resoluções temporais de 0,1ms a $1,0 \mathrm{~ms}$

Tabela 3: Parâmetros $\mathrm{T}_{\mathrm{F}(\max )}, \mathrm{V}_{\mathrm{F}(\max )}$ e $\mathrm{V}_{\mathrm{F}}$ * calculados para os OAP dos pixels + e O, processados por Butterworth e Spline Fit obtendo interpolação para $0,1 \mathrm{~ms}$, para resoluções espaciais de $0,4 \mathrm{~mm}, 0,8 \mathrm{~mm}, 1,2 \mathrm{~mm}, 1,6 \mathrm{~mm}$ e $3,2 \mathrm{~mm}$... 85 Tabela 4: Parâmetros $\mathrm{T}_{\mathrm{F}(\max )}, \mathrm{V}_{\mathrm{F}(\max )}$ e $\mathrm{V}_{\mathrm{F}}$ * calculados para os OAP dos pixels + e $\mathbf{O}$, com resolução espacial de $0,4 \mathrm{~mm}$ e processados por Butterworth e Spline Fit obtendo resoluções temporais de $0,1 \mathrm{~ms}, 0,2 \mathrm{~ms}, 0,3 \mathrm{~ms}, 0,4 \mathrm{~ms}$, $0,5 \mathrm{~ms}$ e $1,0 \mathrm{~ms}$.

Tabela 5: Parâmetros $\mathrm{T}_{\mathrm{S}(\max )}, \mathrm{V}_{\mathrm{S}(\max )} \mathrm{e} \mathrm{V}_{\mathrm{S}}$ * do upstroke dos potenciais de ação simulados para uma célula $\left(\mathrm{AP}_{\text {cell }}\right)$ e para o grupo de 125 células $\left(\mathrm{AP}_{\mathrm{avg}}\right)$, com resolução temporal de $0,1 \mathrm{~ms}$.

Tabela 6: Parâmetros $\mathrm{T}_{\mathrm{S}(\max )}, \mathrm{V}_{\mathrm{S}(\max )} \mathrm{e} \mathrm{V}_{\mathrm{S}}$ do upstroke dos potenciais de ação simulados de $\mathrm{AP}_{\text {cell }}$ com resolução temporal de $0,1 \mathrm{~ms} ; \mathrm{AP}_{\text {avg }}$ com resolução temporal de $0,1 \mathrm{~ms}$ e $2,0 \mathrm{~ms} ; \mathrm{AP}_{\text {avg }}$ com resolução temporal de $2,0 \mathrm{~ms}$ com adição de ruído; e $\mathrm{AP}_{\text {avg }}$ com resolução temporal de 2,0ms e aplicação de processamento digital (filtragem por Butterworth e interpolação por Spline Fit).

Tabela 7: Diferença entre Parâmetros $\mathrm{T}_{\mathrm{F}(\max )}, \mathrm{V}_{\mathrm{F}(\max )}$ e $\mathrm{V}_{\mathrm{F}}$ * calculados para os potenciais de ação ópticos entre os pixels + e $\mathbf{O}$, processados por Butterworth e Spline Fit obtendo resolução temporal de $0,1 \mathrm{~ms}$ para resoluções espaciais de $0,4 \mathrm{~mm}, 0,8 \mathrm{~mm}, 1,2 \mathrm{~mm}, 1,6 \mathrm{~mm}$ e $3,2 \mathrm{~mm}$. 95

Tabela 8: Diferença entre Parâmetros $\mathrm{T}_{\mathrm{F}(\max )}, \mathrm{V}_{\mathrm{F}(\max )}$ e $\mathrm{V}_{\mathrm{F}} *$ calculados para os potenciais de ação ópticos entre os pixels + e $\mathbf{O}$ com resolução espacial de 
0,4mm e processados por Butterworth e Spline Fit obtendo resoluções

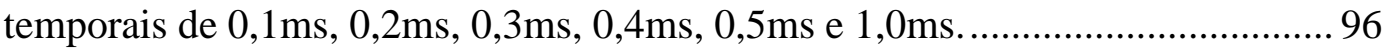
Tabela 9: Variação dos parâmetros eletrofisiológicos para os resultados simulados (A) e experimentais (B), em função da resolução espacial ou temporal. A seta $\downarrow$ indica diminuição do valor e a seta, $\uparrow$ aumento do valor. ...... 97 


\section{Definição dos Símbolos:}

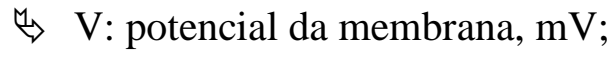

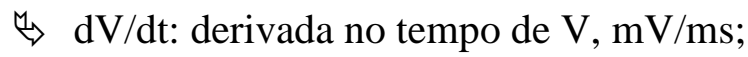

$\stackrel{\leftrightarrow}{\leftrightarrow} \mathrm{I}_{\mathrm{T}}$ : corrente total dos canais iônicos, $\mu \mathrm{A} / \mu \mathrm{F}$;

$\Leftrightarrow \mathrm{I}_{\mathrm{st}}$ corrente do estímulo aplicado, $\mu \mathrm{A} / \mu \mathrm{F}$;

$\Leftrightarrow \mathrm{C}_{\mathrm{m}}$ : capacitância da membrana, $\mu \mathrm{F}$;

$\stackrel{4}{4}$ F: constant de Faraday, 96,500, C/mol;

$\leftrightarrow \mathrm{R}$ : constante de gas, 1,987 calorias $/ \mathrm{mol} /{ }^{\circ} \mathrm{K}$;

$\Leftrightarrow$ T: temperatura, ${ }^{\circ} \mathrm{K}$;

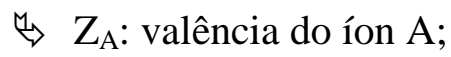

$\stackrel{\mathrm{H}}{\rightarrow}[\mathrm{A}]_{0}$ e $[\mathrm{A}]_{\mathrm{i}}$ : concentrações extracelulares e intracelulares do íon A, respectivamente, $\mathrm{mmol} / \mathrm{L}$;

$\stackrel{E_{\mathrm{A}}}{\mathrm{a}}$ : potencial de Nerst do íon $\mathrm{A}, \mathrm{mV}$;

$\stackrel{\leftrightarrow}{\rightarrow} \mathrm{P}_{\mathrm{A}}$ : permeabilidade da membrana ao íon $\mathrm{A}, \mathrm{cm} / \mathrm{s}$;

$\stackrel{\leftrightarrow}{\rightarrow} \mathrm{P}_{\mathrm{AB}}$ : razão da permeabilidade da membrana ao íon A para íon B;

$\stackrel{\leftrightarrow}{\leftrightarrow} \gamma_{\mathrm{A}}$ : coeficiente de atividade do íon A;

$\stackrel{\leftrightarrow}{\leftrightarrow} \alpha_{y}$ e $\beta_{y}$ : constantes da taxa de abertura e fechamento do portão y, respectivamente, $\mathrm{ms}^{-1}$;

$\stackrel{\leftrightarrow}{\leftrightarrow} \tau_{\mathrm{y}}$ : constante de tempo do portão $\mathrm{y}, \mathrm{ms}$;

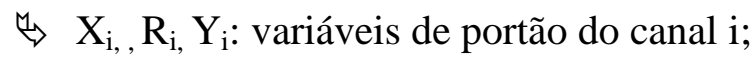

$\leftrightarrow \overline{\mathrm{I}}_{\mathrm{i}}$ : corrente máxima que passa através do canal $\mathrm{i}, \mu \mathrm{A} / \mu \mathrm{F}$;

$\Leftrightarrow \mathrm{G}_{\mathrm{i}}$ condutância do canal i, $\mathrm{mS} / \mu \mathrm{F}$;

$\stackrel{\leftrightarrow}{\rightarrow} \mathrm{K}_{\mathrm{m}, \mathrm{i}}$ : concentração de meia-saturação do canal i, mmol/L;

$\Leftrightarrow \mathrm{I}_{\mathrm{Na}}$ : Corrente de sódio rápida, $\mu \mathrm{A} / \mu \mathrm{F}$;

$\Leftrightarrow$ m: portão de ativação de $\mathrm{I}_{\mathrm{Na}}$;

$\stackrel{\leftrightarrow}{\rightarrow}$ : portão de inativação rápido de $\mathrm{I}_{\mathrm{Na}}$;

$\Leftrightarrow$ j: portão de inativação lento de $\mathrm{I}_{\mathrm{Na}}$;

$\stackrel{\leftrightarrow}{\rightarrow} \mathrm{I}_{\mathrm{Kr}}$ : Corrente de potássio ativada rapidamente, $\mu \mathrm{A} / \mu \mathrm{F}$;

$\Leftrightarrow \mathrm{I}_{\mathrm{Ks}}$ : Corrente de potássio ativada lentamente, $\mu \mathrm{A} / \mu \mathrm{F}$;

$\stackrel{\leftrightarrow}{\rightarrow} \mathrm{I}_{\mathrm{Ca}, \mathrm{L}}$ : Corrente de cálcio que atravessa canais do tipo- $\mathrm{L}, \mu \mathrm{A} / \mu \mathrm{F}$;

$\Leftrightarrow$ d: portão de ativação de $\mathrm{I}_{\mathrm{Ca}, \mathrm{L}}$;

$\Leftrightarrow$ f: portão de inativação (dependente da voltagem) de $\mathrm{I}_{\mathrm{Ca}, \mathrm{L}}$;

$\stackrel{\leftrightarrow}{\rightarrow} \mathrm{f}_{\mathrm{ca}}$ : portão de inativação (dependente da concentração de cálcio) de $\mathrm{I}_{\mathrm{Ca}, \mathrm{L}}$; 
(4) $\mathrm{I}_{\mathrm{Ca}, \mathrm{T}}$ : Corrente de cálcio que atravessa canais do tipo-T, $\mu \mathrm{A} / \mu \mathrm{F}$;

$\Leftrightarrow$ b: portão de ativação de $\mathrm{I}_{\mathrm{Ca}, \mathrm{T}}$;

$\Leftrightarrow$ g: portão de inativação (dependente da voltagem) de $\mathrm{I}_{\mathrm{Ca}, \mathrm{T}}$;

$\Leftrightarrow \mathrm{I}_{\mathrm{K} 1}$ : Corrente de potássio independente do tempo, $\mu \mathrm{A} / \mu \mathrm{F}$;

$\Leftrightarrow \mathrm{K}_{\mathrm{i}}$ : portão de inativação de $\mathrm{I}_{\mathrm{K} 1}$;

$\Leftrightarrow \mathrm{I}_{\mathrm{Kp}}$ : Corrente de potássio de Plateau, $\mu \mathrm{A} / \mu \mathrm{F}$;

$\Leftrightarrow \mathrm{K}_{\mathrm{p}}$ : portão de inativação de $\mathrm{I}_{\mathrm{Kp}}$;

$\Leftrightarrow \mathrm{I}_{\mathrm{NaCaX}}$ : Fluxo pelo trocador sódio-cálcio, $\mu \mathrm{A} / \mu \mathrm{F}$;

$\Leftrightarrow \mathrm{k}_{\mathrm{NaCa}}$ : fator de escala da $\mathrm{I}_{\mathrm{NaCaX}}, \mu \mathrm{A} / \mu \mathrm{F}$;

$\Leftrightarrow \mathrm{k}_{\mathrm{sat}}$ : fator de saturação da $\mathrm{I}_{\mathrm{NaCaX}}$ para potenciais muito negativos;

(4) ๆ: posição da barreira de energia controlando a dependência da voltagem de $\mathrm{I}_{\mathrm{NaCaX}}$

$\Leftrightarrow \mathrm{I}_{\mathrm{NaK}}$ : Corrente da bomba sódio-potássio, $\mu \mathrm{A} / \mu \mathrm{F}$;

$\Leftrightarrow \mathrm{f}_{\mathrm{NaK}}$ : parâmetro dependente da voltage em $\mathrm{I}_{\mathrm{NaK}}$;

$\Leftrightarrow \sigma$ : fator dependente da concentração extracelular de sódio em $\mathrm{I}_{\mathrm{NaK}}$;

$\Leftrightarrow \mathrm{I}_{\mathrm{To}, \mathrm{f}}$ : Corrente transiente de saída rápida, $\mu \mathrm{A} / \mu \mathrm{F}$;

$\Leftrightarrow \mathrm{I}_{\mathrm{To}, \mathrm{s}}$ : Corrente transiente de saída lenta, $\mu \mathrm{A} / \mu \mathrm{F}$;

$\Leftrightarrow \mathrm{I}_{\mathrm{pCa}}$ : Corrente da bomba de cálcio do retículo sarcoplasmático, $\mu \mathrm{A} / \mu \mathrm{F}$;

$\Leftrightarrow \mathrm{I}_{\text {Cab }}$ : Corrente de fundo de cálcio, $\mu \mathrm{A} / \mu \mathrm{F}$;

$\Leftrightarrow \mathrm{I}_{\mathrm{Nab}}$ : Corrente de fundo de sódio, $\mu \mathrm{A} / \mu \mathrm{F}$;

$\leftrightarrow \mathrm{I}_{\text {rel: }}$ Corrente de liberação de cálcio do retículo sarcoplasmático de junção (JSR), mmol/L.ms;

(4) $\mathrm{G}_{\text {rell }}$ taxa máxima de liberação de cálcio do JSR, ms;

$\Leftrightarrow \mathrm{I}_{\text {up: }}$ : Corrente de entrada de cálcio no retículo sarcoplasmático em rede (NSR), mmol/L.ms;

$\Leftrightarrow \mathrm{I}_{\text {leak }}$ : Corrente de vazamento de cálcio do retículo sarcoplasmático em rede (NSR), mmol/L.ms;

$\Leftrightarrow \mathrm{K}_{\text {leak }}:$ taxa de vazamento de cálcio do NSR, $\mathrm{ms}^{-1}$;

$\Leftrightarrow \mathrm{I}_{\mathrm{tr}}$ : Corrente de tranferência de cálcio do retículo sarcoplasmático em rede (NSR) para o retículo sarcoplasmático de junção (JSR), mmol/L.ms;

$\Leftrightarrow \mathrm{R}_{\mathrm{y}} \mathrm{R}_{\text {open }}$ : taxa de abertura de canais de liberação de cálcio do JSR.

$\Leftrightarrow \mathrm{R}_{y} \mathrm{R}_{\text {close: }}$ taxa de fechamento de canais de liberação de cálcio do JSR. 
"Still round the corner there may wait, a new road or a secret gate."

J. R. R. Tolkien 